\title{
Details des Klinik-Entlassmanagements geregelt
}

_ Alle Details zum neuen Entlassmanagement, an das sich die Krankenhäuser ab Juli 2017 bei jedem Patienten halten müssen, sind nach einer Entscheidung des erweiterten Bundesschiedsamts vom 13. Oktober 2016 nunmehr festgelegt worden. Das Schiedsamt war Ende Juni angerufen worden, nachdem Kliniken, Kassen und KBV die Verhandlungen für gescheitert erklärt hatten. $\mathrm{Zu}$ dem Zeitpunkt lag zwar bereits ein weitgehend konsentierter Rahmenvertrag vor, einige zentrale Punkte waren aber noch offen.

\section{MMW-KOMMENTAR}

Der Rahmenvertrag umfasst alle Patienten, die voll-oder teilstationär behandelt werden.
Somit haben künftig alle Patienten gegenüber dem Krankenhaus Anspruch auf ein Entlassmanagement inklusive Entlassgespräch und Entlassbrief.

Die Krankenkasse ist verpflichtet, den Prozess bei Bedarf zu unterstützen, wenn der Patient zustimmt. Hierbei geht es vor allem um genehmigungspflichtige Leistungen wie häusliche Krankenpflege, Hilfsmittel oder Maßnahmen zur Rehabilitation, die möglichst beantragt und genehmigt werden sollen, noch bevor der Patient entlassen wird.

Krankenhäuser dürfen künftig für einen begrenzten Zeitraum Arznei-, Verband-, Heilund Hilfsmittel, häusliche Krankenpflege sowie Soziotherapie verordnen und die Arbeitsunfähigkeit feststellen. Ferner wurde entschieden, dass der Medikationsplan bei der
Entlassung den Vorgaben des § $31 a$ SGB V entsprechen soll. Er wird demnach den gleichen Linien folgen wie die Medikationspläne der ambulanten Praxen.

Die Krankenhäuser sind verpflichtet, die weiterbehandelnden Ärzte so früh wie möglich einzubeziehen. Unter anderem muss ein Ansprechpartner für das Entlassmanagement montags bis freitags von 9-19 Uhr sowie am Wochenende von 10-14 Uhr telefonisch für Rückfragen zur Verfügung stehen. Pflicht ist neben dem Entlassgespräch auch ein Entlassbrief. Schon am Tag der Entlassung erhält der Patient zumindest eine vorläufige Version. Mit seiner Einwilligung erhalten auch der die Anschlussversorgung durchführende Arzt und ggf. der einweisende Arzt eine Kopie.

\section{Neues DMP Herzinsuffizienz wird eingeführt}

_ Der Gemeinsame Bundesausschuss (G-BA) hat ein Disease-ManagementProgramm (DMP) für Patienten mit chronischer Herzinsuffizienz auf den Weg gebracht. Danach wird das derzeit noch bestehende Modul Herzinsuffizienz aus dem DMP Koronare Herzkrankheit (KHK), das im Jahr 2009 eingeführt wurde, in ein eigenes DMP Herzinsuffizienz überführt.

\section{MMW-KOMMENTAR}

Der Beschluss zum neuen DMP Herzinsuffizienz wird voraussichtlich im August 2017 gefasst. Das Zentralinstitut für die kassenärztliche Versorgung (Zi) hatte in einer Studie belegt, dass Patienten mit chronischen Erkrankungen wie einer Herzinsuffizienz von einer intensiven ambulanten Behandlung profitieren. Mit knapp 433.000 stationär behandelten Herzinsuffizienz-Patienten im Jahr 2014 ist die Erkrankung dem Zi zufolge nun der zweithäufigste stationäre Aufnahmegrund nach der Schwangerschaft. Darüber hinaus hat der G-BA die Aktualisierung der DMP für Diabetes mellitus Typ 1 und $K H K$ beschlossen. Zwei weitere strukturierte Behandlungsprogramme, nämlich für Depression und Rückenschmerz, hat der Gesetzgeber mit dem Versorgungsstärkungsgesetz angestoßen. Auch diese sollen noch bis Jahresende entwickelt werden.

Bislang gibt es bundesweit Behandlungsprogramme für Asthma bronchiale, COPD, Diabetes mellitus Typ 1 und 2, KHK und Brustkrebs. Seit Einführung der DMP vor mehr als zehn Jahren sind die Teilnehmerzahlen kontinuierlich gestiegen. Aktuell nehmen über 6,5 Millionen Patienten teil. Daran hat das DMP Typ-2-Diabetes mit gut 4 Millionen den mit Abstand größten Anteil. Knapp 1,8 Millionen Patienten sind in das DMP KHK eingeschrieben, knapp 900.000 ins DMP Asthma und gut 700.000 ins DMP COPD.

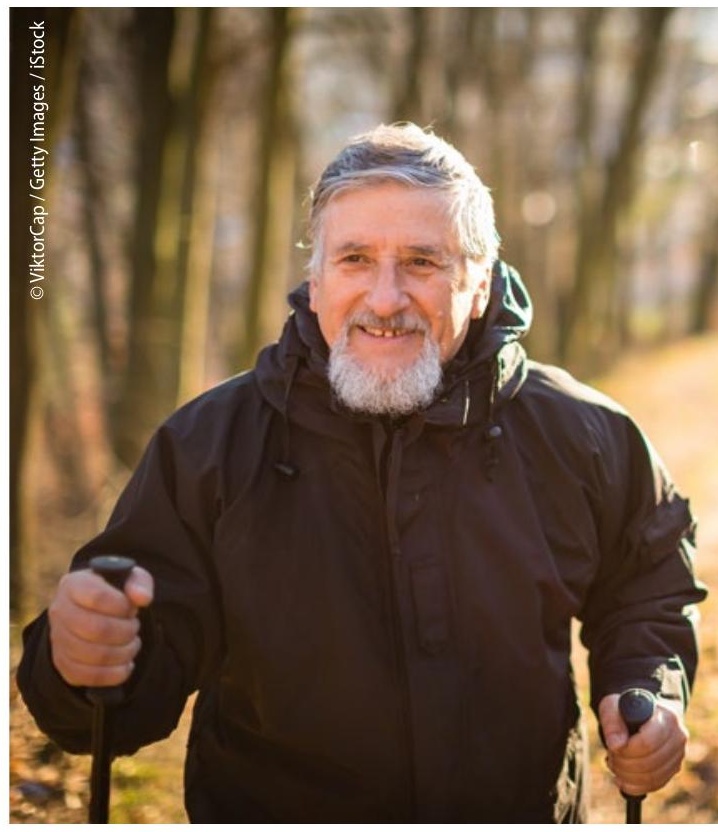

Herzinsuffiziente Patienten erhalten ein strukturiertes Behandlungsprogramm. 